\title{
Association between Maternal Obesity Class, Adherence to Labor Guidelines, and Perinatal Outcomes
}

\author{
Irene A. Stafford, $\mathrm{MD}^{1}$ \\ Angela Burgess, $\mathrm{MD}^{1}$ Mark Turrentine, $\mathrm{MD}^{3}$ \\ ${ }^{1}$ Department of Obstetrics and Gynecology, University of Texas \\ Health Science Center, Houston, Texas \\ 2 Department of Obstetrics and Gynecology, Hurley Medical Center, \\ Michigan State University, Flint, Michigan \\ ${ }^{3}$ Department of Obstetrics and Gynecology, Baylor College of \\ Medicine, Houston, Texas
}

Lauren Spoo, $\mathrm{MD}^{3} \quad$ Alexandra Berra, $\mathrm{MD}^{3}$

\begin{abstract}
Address for correspondence Irene A. Stafford, MD, Department of Obstetrics and Gynecology, Baylor College of Medicine, 6431, Fannin, MSB3.286, Houston, TX 77030 (e-mail: Irene.Stafford@bcm.edu).
\end{abstract}

AJP Rep 2021;11:e105-e112.

\begin{abstract}
Keywords

- overweight

- obesity

- labor dystocia

- primary cesarean guidelines

Background Data are limited concerning rates of perinatal complications in women with a body mass index (BMI) $\geq 40 \mathrm{~kg} / \mathrm{m} 2$ compared to women with other BMI classes when guidelines for the safe prevention of the primary cesarean delivery are applied. Objective The aim of the study is to evaluate labor guideline adherence by BMI class and to compare perinatal outcomes across BMI classes with guideline adherent management.

Study Design This retrospective study included low-risk women admitted for delivery between April 2014 and April 2017 after the labor guidelines were implemented. BMI closest to delivery was used for analysis. Women with cesarean for nonreassuring fetal status were excluded.

Results Guideline adherence decreased with increasing BMI, with 93\% adherence among women of normal weight compared to $81 \%$ for class III obese women $(p<0.0001)$. Among women who had guideline-adherent management, there was increased rates of cesarean among class III versus other obesity classes; however, there were no differences in rates of infectious morbidity $(p=0.98)$ or hemorrhage $(p=0.93)$. Although newborns of women with class III obesity had higher rates of meconium at birth, neonatal outcomes were not different with increasing maternal BMI $(p=0.65)$.

Conclusion There were no differences in adverse perinatal outcomes with increasing BMI.
\end{abstract}

Obesity, defined by the Word Health Organization as BMI $\geq 30 \mathrm{~kg} / \mathrm{m} 2$ (BMI = weight $[\mathrm{kg}] /$ height $[\mathrm{m}]$ squared) is currently the most common medical condition among reproductive aged (23-39 years old) women. ${ }^{1,2}$ Obese pregnant women have higher rates of antepartum comorbidities, such as gestational diabetes or hypertensive disorders, and often experience iatrogenic preterm birth and increased peripartum complications such as postpartum hemorrhage, received

January 12, 2020 accepted after revision April 6, 2020
DOI https://doi.org/ $10.1055 / \mathrm{s}-0041-1732409$. ISSN 2157-6998.

\footnotetext{
(C) 2021. The Author(s).

This is an open access article published by Thieme under the terms of the Creative Commons Attribution-NonDerivative-NonCommercial-License, permitting copying and reproduction so long as the original work is given appropriate credit. Contents may not be used for commercial purposes, or adapted, remixed, transformed or built upon. (https://creativecommons.org/ licenses/by-nc-nd/4.0/)

Thieme Medical Publishers, Inc., 333 Seventh Avenue, 18th Floor, New York, NY 10001, USA
} 
thromboembolic events, and infectious morbidity with increasing weight class. ${ }^{3-21}$ The odds ratios of cesarean delivery in overweight and obese women are 1.4 and 2.0, respectively when compared to their normal weight counterparts. $^{4,22}$ Similarly, neonatal morbidity and mortality is significantly increased with increasing maternal BMI. ${ }^{6-9,23-26}$ Recent data suggest that prepregnancy BMI is linked to higher neonatal intensive care admissions, lower Apgar scores, and a relative risk of 1.15 for neonatal death. ${ }^{8,9,23-26}$ Although these studies demonstrate similar trends in adverse maternal and perinatal outcomes, they remain limited in their evaluation of the effect of labor duration on the aforementioned complications. ${ }^{4,9-15}$

Evidence suggests that the length of active labor is longer in overweight and obese women, even when adjusting for confounding variables such as medical or antenatal comorbidities, gestational age at delivery, birthweight, and induction. ${ }^{9-12}$ It has been demonstrated that in obese women, the rate of cervical dilation is inversely associated with maternal weight and that for each $10-\mathrm{kg}$ increase, the rate of dilation decreased by $0.04 \mathrm{~cm} / \mathrm{h} .{ }^{11}$ Yet other studies have shown that the median length of labor is not associated with maternal BMI. 47-20,25,27-29 Importantly, these analyses do not address adherence by providers to contemporary guidelines for management of protracted labor and the prevention of the primary cesarean delivery. ${ }^{17,21,24,30}$ The American College of Obstetricians and Gynecologists support the extension of duration of labor in obese patients in order to avoid cesarean delivery and associated complications. ${ }^{17}$ However, the increased risk of adverse perinatal outcomes in this patient population with prolonged labor should be taken into consideration, with the outcomes analyzed and compared when applied to current practices for the prevention of the primary cesarean delivery. ${ }^{17,24,30}$ The aim of this study was to evaluate provider labor guideline adherence with BMI class and to determine differences in maternal and neonatal outcomes with guideline adherent labor management across BMI weight class.

\section{Materials and Methods}

This retrospective cohort study included all low-risk women with a singleton, term (37 ${ }^{0 / 7}$ to $40^{6 / 7}$ weeks), cephalic, pregnancy admitted in active labor $(\geq 5$-cm cervical dilation in the presence of regular contractions) or undergoing labor induction between April 30, 2014 through April 30, 2017, at Ben Taub Hospital and the Texas Children's Pavilion for Women after the prevention of the primary cesarean delivery and labor dystocia guidelines, ${ }^{21,24,30}$ were implemented by the Department of Obstetrics and Gynecology at the Baylor College of Medicine (Houston, TX) for the management of labor. The protocol includes the support of evidencebased options for cervical ripening followed by the intrapartum management of latent labor. ${ }^{21,30}$

A failed labor course may be defined in two ways: first, failed induction with rupture of membranes greater than 18 hours with the use of oxytocin augmentation over 24 hours, or second, as an arrest disorder, dilation of $6 \mathrm{~cm}$ or greater with membrane rupture and no cervical change for 4 hours or more of adequate contractions, or 6 hours or more if contractions are inadequate resulting in a cesarean delivery. ${ }^{21,24}$ In addition, 3 hours without epidural, or 4 hours with epidural in a nulliparous patient or 2 hours without epidural, or 3 hours with epidural with a multiparous patient and pushing at complete cervical dilation without a vaginal birth is required before a cesarean for arrest of descent is determined. $^{21,24}$

Patients included in the study were identified using the PeriBank database. PeriBank is an Institutional Review Boardapproved comprehensive, prospectively recorded, institutional database and biobank focusing on detailed clinical data and accompanying specimens collected at delivery and curated at Baylor College of Medicine, Houston, TX. A detailed description of PeriBank has been previously published. ${ }^{31}$ The database was queried for demographic, delivery, maternal, and neonatal outcome data including, indication for induction, length of labor (all stages), membrane rupture, oxytocin administration, and indication for cesarean in accordance with the Strengthening the Reporting of Observational Studies in Epidemiology guidelines (STROBE). ${ }^{32}$ The index pregnancy was the most recent pregnancy in the database for each subject and the BMI closest to delivery was used for analysis for consistency due to variation in gestational age in presentation for care. Pregnancies complicated by fetal anomalies and women with a contraindication to a vaginal delivery (malpresentation, abnormal placentation, and prior myomectomy) were excluded. In addition, women with a BMI $<18.5$, pregestational diabetes, hypertensive disorders of pregnancy antepartum stillbirth, fetal growth restriction (defined as estimated fetal weight below the 10th percentile for gestational age) prior cesarean delivery and cesarean for nonreassuring fetal status in this index pregnancy were excluded to minimize factors that may confound results.

The primary outcome was provider adherence to the published labor dystocia guidelines across BMI classes. In addition, a comparison of maternal and neonatal outcomes when labor management was compliant with published guidelines across groups was analyzed. Maternal variables collected included gestational age at delivery, labor type (scheduled induction of labor, labor augmentation for patients presenting in labor or spontaneous labor), mode of delivery, method of anesthesia used, oligohydramnios (defined as maximum vertical pocket less than $2 \mathrm{~cm}$, or amniotic fluid index less than $5 \mathrm{~cm}$ ), chorioamnionitis, endomyometritis, hemorrhage ( $>1,000 \mathrm{~mL}$ blood loss for cesarean delivery and vaginal delivery), and a composite of adverse maternal outcomes defined as blood transfusion, hysterectomy, wound infection, wound separation, ileus and delayed postpartum hemorrhage ( $>24$ hours after delivery).

Neonatal characteristics obtained were newborn weight, Apgar scores, meconium passage at birth, arterial blood gas, neonatal intensive care unit admission for sepsis evaluation and a composite of adverse outcomes defined as respiratory distress, transient tachypnea of the newborn, necrotizing enterocolitis, bronchopulmonary dysplasia, retinopathy, pulmonary interstitial emphysema, hypertonicity, or use of pressors. BMI classes in $\mathrm{kg} / \mathrm{m}^{2}$ were defined as follows: normal 
weight 18.5 to $<25$, overweight 25 to $<30$, class I obesity 30 to $<35$, class II obesity 35 to $<40$, and class III obesity $\geq 40$.

Protocol adherence was considered if a cesarean delivery occurred for a failed labor or induction course as previously defined using recorded times for admission, initiation of active labor, rupture of membranes, oxytocin augmentation, Montevideo units, labor stages, and delivery as collected in Peribank..$^{21,24}$ Residents, midwives, obstetrician-gynecologists, and maternal fetal medicine providers manage labor and delivery units at both sites. All decisions concerning labor dystocia and cesarean delivery are made with agreement between house staff and faculty.

Descriptive statistics were calculated for all study variables. Women were grouped according to their BMI class and compared by the Kruskal-Wallis for continuous or ordinal outcomes and chi-square/Fisher exact test for categorical outcomes. Logistic regression of guideline adherence was conducted controlling for maternal age, race, ethnicity, parity, and newborn weight. Subgroup analyses were conducted in women who underwent induction. In this subgroup, logistic and generalized linear regression of maternal and neonatal outcomes was used to determine if there was an interaction between gestational age at delivery and BMI. The regression model controlled for the potential confounders of age, race, Hispanic ethnicity, parity, and newborn weight. $p<0.05$ was considered significant and no adjustment was made for multiple comparisons. All analyses were performed in SAS software (9.4 SAS Institute Inc, Cary, NC).

\section{Results}

There were 13,978 deliveries post implementation of the labor dystocia guidelines at both delivery sites during the study period. Of these, 949 were missing data on BMI or had a BMI $<18.5$ and were excluded, leaving a total of 13,029 deliveries for analysis (See Flow diagram - Fig. 1). Guideline adherence decreased with increasing BMI, with 93\% adherence among women of normal weight compared to $89 \%$ for class I, $88 \%$ for class II, and $81 \%$ for class III obese women $(p<0.0001$ adjusted for race, parity, neonatal weight, and maternal age; - Table 1). Demographic and baseline characteristics of the population treated in accordance with guidelines are presented in -Table $\mathbf{2}$. There was no clinically significant difference in maternal age, race, ethnicity, and parity across the BMI classes; however, the percentage of Hispanic women increased with increasing BMI. There were more inductions of labor and cesarean deliveries with increasing weight class; however, there was no difference in rates of chorioamnionitis, endomyometritis, hemorrhage, or the maternal composite outcome after adjusting for parity, maternal age, race, and Hispanic ethnicity $(p=0.98,0.63$, and 0.09 , respectively). These results are demonstrated in - Table 3.

Neonatal outcomes are shown in -Table 4. Newborn birthweight increased significantly with increasing maternal BMI and newborns of women with class III obesity had higher rates of meconium passage at birth $(p<0.001)$. However, a composite of neonatal outcomes was not different with increasing maternal BMI $(p=0.65)$. When analyzing the subgroup of women who underwent induction $(n=2,658)$, there was no difference in the number of women induced at different gestational ages across BMI classes $(p=0.92)$ and the relationships between BMI class and chorioamnionitis, endomyometritis, and sepsis were not different across the gestational age categories ( $p$-value is for interaction term, $p=0.60,0.30,0.70$, respectively) when adjusting for age,
*Inclusion Criteria

Absence of:

- Cervical dilation $<5 \mathrm{~cm}$ without regular contractions

Antenatal complications

Hypertensive disorders of pregnancy

Gestational or pregestational diabetes

Medical problems

Fetal anomalies

Fetal growth restriction

Contraindications to vaginal delivery

Non-reassuring fetal tracing
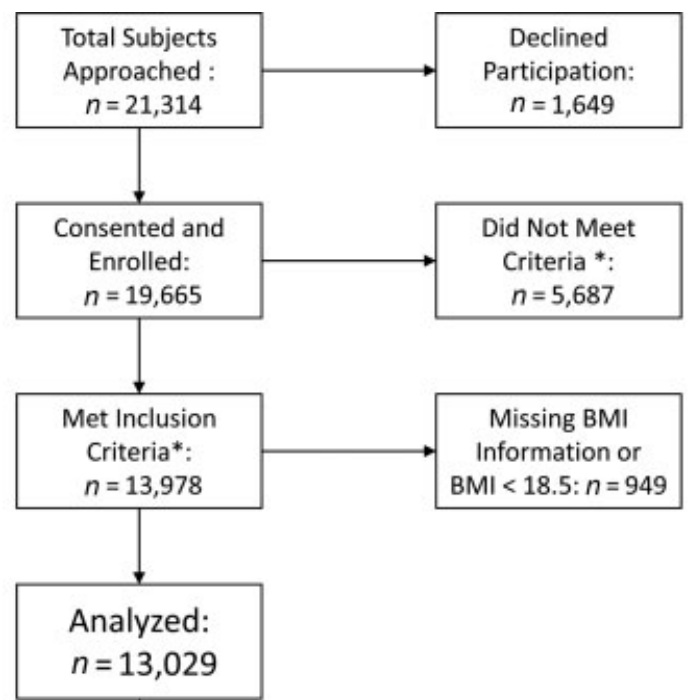

Analyzed.

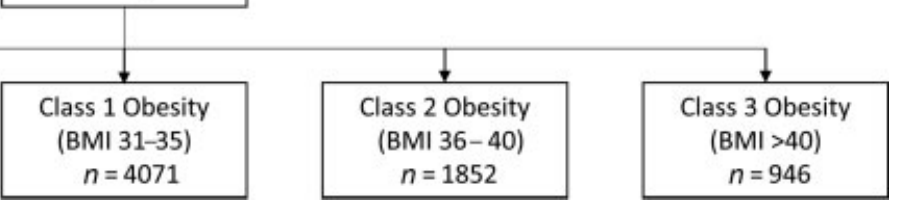

Fig. 1 Association between maternal obesity class, adherence to labor guidelines, and perinatal outcomes. Patients consented and enrolled April 30, 2014 to April 30, 2017. 
Table 1 Guideline adherence by BMI category

\begin{tabular}{|l|l|l|l|l|l|l|}
\hline & $\begin{array}{l}\text { Normal weight BMI } \\
\geq 18.5 \text { to }<25 \\
(N=1,361)\end{array}$ & $\begin{array}{l}\text { Overweight BMI } \\
\mathbf{2 5} \text { to }<30 \\
(N=4,799)\end{array}$ & $\begin{array}{l}\text { Class 1 obesity BMI } \\
\begin{array}{l}30 \text { to }<35 \\
(N=4,071)\end{array}\end{array}$ & $\begin{array}{l}\text { Class 2 obesity BMI } \\
35 \text { to }<40 \\
(N=1,852)\end{array}$ & $\begin{array}{l}\text { Class 3 obesity } \\
\text { BMI } \geq 40 \\
(N=946)\end{array}$ & $\begin{array}{l}\text { Total } \\
(N=13,029)\end{array}$ \\
\hline $\begin{array}{l}\text { Did not follow } \\
\text { guidelines }\end{array}$ & $101(7.4 \%)$ & $435(9.1 \%)$ & $448(11.0 \%)$ & $218(11.8 \%)$ & $184(19.5 \%)$ & $1,386(10.6 \%)$ \\
\hline $\begin{array}{l}\text { Followed } \\
\text { guidelines }\end{array}$ & $1,260(92.6 \%)$ & $4,364(90.9 \%)$ & $3,623(89.0 \%)$ & $1,634(88.2 \%)$ & $762(80.6 \%)$ & $1,1643(89.4 \%)$ \\
\hline
\end{tabular}

Note: $p \leq 0.0001$ both unadjusted and adjusted for white race, parity, neonatal weight, and maternal age; data presented as $N(\%)$.

Table 2 Demographics and baseline characteristics (guideline followed)

\begin{tabular}{|c|c|c|c|c|c|}
\hline & $\begin{array}{l}\text { Normal weight } \\
\text { BMI } \geq 18.5 \text { to } \\
<25(N=1,260)\end{array}$ & $\begin{array}{l}\text { Overweight } \\
\text { BMI } 25 \text { to }<30 \\
(N=4,364)\end{array}$ & $\begin{array}{l}\text { Class } 1 \text { obesity } \\
\text { BMI } 30 \text { to }<35 \\
(N=3,623)\end{array}$ & $\begin{array}{l}\text { Class } 2 \text { obesity } \\
\text { BMI } 35 \text { to }<40 \\
(N=1,634)\end{array}$ & $\begin{array}{l}\text { Class } 3 \text { obesity } \\
\text { BMI } \geq 40 \\
(N=762)\end{array}$ \\
\hline \multicolumn{6}{|l|}{ Age } \\
\hline Mean (SD) & $27.6(6.07)$ & $29.1(5.84)$ & $29.3(5.77)$ & $29.2(5.82)$ & $29.1(5.62)$ \\
\hline \multicolumn{6}{|l|}{ Race } \\
\hline Black & $157(12.5 \%)$ & $544(12.5 \%)$ & $491(13.6 \%)$ & $247(15.1 \%)$ & $178(23.4 \%)$ \\
\hline Caucasian & $950(75.4 \%)$ & $3,449(79.0 \%)$ & $2,929(80.8 \%)$ & $1,328(81.3 \%)$ & $567(74.4 \%)$ \\
\hline Asian & $123(9.8 \%)$ & $292(6.7 \%)$ & $139(3.8 \%)$ & $22(1.4 \%)$ & $12(1.8 \%)$ \\
\hline Alaskan or American Indian & $1(0.08 \%)$ & $1(0.02 \%)$ & $1(0.03 \%)$ & $1(0.06 \%)$ & $2(0.26 \%)$ \\
\hline Hawaiian or Pacific Islander & $3(0.24 \%)$ & $1(0.02 \%)$ & $1(0.03 \%)$ & 0 & $2(0.26 \%)$ \\
\hline Unknown & $31(2.5 \%)$ & $92(2.1 \%)$ & $72(2.0 \%)$ & $38(2.3 \%)$ & $9(1.2 \%)$ \\
\hline \multicolumn{6}{|l|}{ Ethnicity } \\
\hline Hispanic & $548(43.5 \%)$ & $2,217(50.8 \%)$ & $2,236(61.7 \%)$ & $1,090(66.7 \%)$ & $476(62.5 \%)$ \\
\hline Not Hispanic & $712(56.5)$ & $2,146(49.2)$ & $1,385(38.2)$ & $544(33.3)$ & $286(37.5)$ \\
\hline Not disclosed & 0 & $1(0.02 \%)$ & $2(0.06 \%)$ & 0 & 0 \\
\hline \multicolumn{6}{|l|}{ BMI } \\
\hline Mean (SD) & $23.4(1.20)$ & $27.6(1.40)$ & $32.2(1.42)$ & $37.1(1.39)$ & $44.2(5.70)$ \\
\hline Gravida & $2(1-3)$ & $2(1-3)$ & $2(2-4)$ & $3(2-4)$ & \\
\hline Parity & $1(0-1)$ & $1(0-2)$ & $1(0-2)$ & $1(0-2)$ & $1(0-2)$ \\
\hline \multicolumn{6}{|l|}{ Marital status } \\
\hline Married & $888(70.5 \%)$ & $3,082(70.6 \%)$ & $2,431(67.1 \%)$ & $\begin{array}{l}1,075 \\
(65.8 \%)\end{array}$ & $472(61.9 \%)$ \\
\hline Single & $332(26.4 \%)$ & $1,134(26.0 \%)$ & $1,064(29.4 \%)$ & $490(30.0 \%)$ & $258(33.9 \%)$ \\
\hline Not reported & $20(1.6 \%)$ & $56(1.3 \%)$ & $52(1.4 \%)$ & $30(1.8 \%)$ & $9(1.2 \%)$ \\
\hline GA at 1st prenatal visit & $11.7(8.7-16.0)$ & $11.4(8.7-15.9)$ & $11.7(8.7-16.0)$ & $11.9(8.9-15.9)$ & $12.0(9.8-18)$ \\
\hline Number of prenatal visits & $11(8-12)$ & $11(9-13)$ & $11(9-13)$ & $11(9-13)$ & $11(8-12)$ \\
\hline HIV & $2(0.16 \%)$ & $13(0.30 \%)$ & $16(0.44 \%)$ & $3(0.18 \%)$ & $6(0.79 \%)$ \\
\hline Hepatitis B & $10(0.79 \%)$ & $23(0.53 \%)$ & $26(0.72 \%)$ & $7(0.43 \%)$ & $6(0.79 \%)$ \\
\hline GBS & $299(23.7 \%)$ & $926(21.2 \%)$ & 795 (21.9\%) & $383(23.4 \%)$ & $217(28.5 \%)$ \\
\hline RPR & $6(0.48 \%)$ & $14(0.32 \%)$ & $8(0.22 \%)$ & $8(0.49 \%)$ & $8(1.1 \%)$ \\
\hline Current smoking & $9(0.71 \%)$ & $23(0.53 \%)$ & $14(0.39 \%)$ & $11(0.67 \%)$ & $9(1.2 \%)$ \\
\hline Prior alcohol use & $25(2.0 \%)$ & $61(1.4 \%)$ & $39(1.1 \%)$ & $15(0.92 \%)$ & $7(0.92 \%)$ \\
\hline Substance use & 0 & $3(0.07 \%)$ & $2(0.06 \%)$ & $1(0.06 \%)$ & 0 \\
\hline
\end{tabular}

Abbreviations: BMI, body mass index; GA, gestational age; GBS, group B Streptococcus; HIV, human immunodeficiency virus; RPR, rapid plasma reagin; SD, standard deviation.

Note: Data presented as $N(\%)$ mean (SD) or median (interquartile range). 
Table 3 Maternal outcomes (guideline followed)

\begin{tabular}{|c|c|c|c|c|c|c|}
\hline & $\begin{array}{l}\text { Normal weight } \\
\mathrm{BMI} \geq 18.5 \text { to }<25 \\
(N=1,260)\end{array}$ & $\begin{array}{l}\text { Overweight BMI } \\
25 \text { to }<30 \\
(N=4,364)\end{array}$ & $\begin{array}{l}\text { Class } 1 \text { obesity } \\
\text { BMI } 30 \text { to }<35 \\
(N=3,623)\end{array}$ & $\begin{array}{l}\text { Class } 2 \text { obesity } \\
\text { BMI } 35 \text { to }<40 \\
(N=1,634)\end{array}$ & $\begin{array}{l}\text { Class } 3 \text { obesity } \\
\text { BMI } \geq 40 \\
(N=762)\end{array}$ & $p$-Value \\
\hline \multicolumn{7}{|l|}{ GA at delivery } \\
\hline Mean (SD) & $39.3(1.09)$ & $39.5(1.07)$ & $39.5(1.12)$ & $39.4(1.09)$ & $39.4(1.09)$ & 0.001 \\
\hline Type of labor & & & & & & $<0.001$ \\
\hline $\begin{array}{l}\text { Spontaneous/ } \\
\text { Augmented }\end{array}$ & $1,013(80.4 \%)$ & $3,433(78.7 \%)$ & 2,775 (76.6\%) & $1,223(74.9 \%)$ & 521 (68.4\%) & \\
\hline Induced/No labor & $244(19.4 \%)$ & $922(21.1 \%)$ & $839(23.2 \%)$ & 407 (24.9\%) & 241 (31.6\%) & \\
\hline Missing & $3(0.24 \%)$ & $9(0.21 \%)$ & $9(0.25 \%)$ & $4(0.24 \%)$ & 0 & \\
\hline Delivery route & & & & & & 0.007 \\
\hline Vaginal & $1,211(96.1 \%)$ & $4,228(96.9 \%)$ & $3,508(96.8 \%)$ & $1,589(97.3 \%)$ & 731 (95.9\%) & \\
\hline $\begin{array}{l}\text { Operative assisted } \\
\text { vaginal }\end{array}$ & $45(3.6 \%)$ & $117(2.7 \%)$ & $93(2.6 \%)$ & $39(2.4 \%)$ & $18(2.4 \%)$ & \\
\hline Cesarean & $4(0.32 \%)$ & $19(0.44 \%)$ & $22(0.61 \%)$ & $6(0.37 \%)$ & $13(1.7 \%)$ & \\
\hline Oligohydramnios & $29(2.3 \%)$ & $101(2.3 \%)$ & $75(2.1 \%)$ & $42(2.6 \%)$ & $27(3.5 \%)$ & 0.18 \\
\hline Anesthesia & 1,077 (85.5\%) & $3,726(85.4 \%)$ & $3,136(86.6 \%)$ & $1,427(87.3 \%)$ & $663(87.0 \%)$ & 0.24 \\
\hline Regional & $885(70.2 \%)$ & $3,009(69.0 \%)$ & $2,556(70.6 \%)$ & $1,209(74.0 \%)$ & 568 (74.5\%) & \\
\hline General & $7(0.56 \%)$ & $22(0.50 \%)$ & $10(0.28 \%)$ & $8(0.49 \%)$ & $8(1.1 \%)$ & \\
\hline Other & $593(47.1 \%)$ & $2,100(48.1 \%)$ & $1,844(50.9 \%)$ & $788(48.2 \%)$ & 390 (51.2\%) & \\
\hline Chorioamnionitis & $54(4.3 \%)$ & $178(4.1 \%)$ & $146(4.0 \%)$ & $69(4.2 \%)$ & $29(3.8 \%)$ & 0.98 \\
\hline Endomyometritis & $5(0.40 \%)$ & $10(0.23 \%)$ & $13(0.36 \%)$ & $5(0.31 \%)$ & $4(0.52 \%)$ & 0.63 \\
\hline Hemorrhage & $40(3.2 \%)$ & $125(2.9 \%)$ & $108(3.0 \%)$ & $47(2.9 \%)$ & $19(2.5 \%)$ & 0.93 \\
\hline Composite $^{a}$ & $14(1.1 \%)$ & $38(0.87 \%)$ & $22(0.61 \%)$ & $12(0.73 \%)$ & $1(0.13 \%)$ & 0.09 \\
\hline Blood transfusion & $11(0.87 \%)$ & $31(0.71 \%)$ & $19(0.52 \%)$ & $9(0.55 \%)$ & 0 & \\
\hline $\begin{array}{l}\text { Hysterectomy from } \\
\text { atony }\end{array}$ & 0 & $1(0.02 \%)$ & $4(0.11 \%)$ & 0 & 0 & \\
\hline Accreta hysterectomy & 0 & 0 & $2(0.06 \%)$ & 0 & 0 & \\
\hline Wound infection & $1(0.08 \%)$ & 0 & 0 & 0 & 0 & \\
\hline Wound separation & 0 & 0 & 0 & 0 & 0 & \\
\hline Ileus & $1(0.08 \%)$ & 0 & 0 & 0 & 0 & \\
\hline $\begin{array}{l}\text { Delayed postpartum } \\
\text { hemorrhage }\end{array}$ & $3(0.24 \%)$ & $11(0.25 \%)$ & $6(0.17 \%)$ & $3(0.18 \%)$ & $1(0.13 \%)$ & \\
\hline
\end{tabular}

Abbreviations: BMI, body mass index; SD, standard deviation.

Note: Data presented as N(\%) or mean (SD) except where indicated.

aBlood transfusion, hysterectomy from atony, accreta hysterectomy, wound infection, wound separation, ileus, delayed postpartum hemorrhage $p$ value for categorical variables from chi-squared test and for continuous variables from Kruskal-Wallis test across BMI categories.

race, ethnicity, parity, and birthweight, despite increasing cesarean rates. When comparing gestational age 37 to 38 to 39 to 40 weeks, there remained no differences in other adverse maternal or neonatal outcomes $(p=0.58,0.51)$. Maternal and neonatal composite outcomes did not differ across BMI categories at separate gestational ages at induction as well ( $p=0.98,0.90$, respectively).

\section{Discussion}

This study demonstrates a decrease in guideline adherence by providers as maternal weight class increased, with the lowest rate of compliance among women with class III obesity. This finding may be a result of bias or weight discrimination that undoubtedly exists in our society and unfortunately, in medicine. In 2018, the American Medical Association Journal of Medical Ethics published research surveying first year medical students for their perception of obesity. Over 70\% showcased negative attitudes toward obesity, with $74 \%$ reporting that they believe obesity is a result of ignorance. ${ }^{33,34}$ Negative weight bias has been shown to adversely affect clinical practice and patient outcomes, including reduced access to surgery for obese patients. ${ }^{19,34,35}$ These preconceived attitudes are often a result of educational deficiencies; however, can translate to prejudice in care, further perpetuating negative health outcomes for obese patients. ${ }^{33}$

The secondary aim was to estimate more accurate perinatal risks for this cohort of women when standardized induction 
Table 4 Neonatal outcomes (guideline followed)

\begin{tabular}{|c|c|c|c|c|c|c|}
\hline & $\begin{array}{l}\text { Normal weight } \\
\text { BMI } \geq 18.5 \text { to }<25 \\
(N=1,260)\end{array}$ & $\begin{array}{l}\text { Overweight BMI } \\
25 \text { to }<30 \\
(N=4,364)\end{array}$ & $\begin{array}{l}\text { Class } 1 \text { obesity BMI } \\
30 \text { to }<35 \\
(N=3,623)\end{array}$ & $\begin{array}{l}\text { Class } 2 \text { obesity } \\
\text { BMI } 35 \text { to }<40 \\
(N=1,634)\end{array}$ & $\begin{array}{l}\text { Class } 3 \text { obesity } \\
\text { BMI } \geq 40 \\
(N=762)\end{array}$ & $p$-Value \\
\hline \multicolumn{7}{|l|}{ Birthweight (g) } \\
\hline Mean (SD) & $3,194.2(373.00)$ & $3,323.5(398.28)$ & $3,397.3(400.58)$ & $3,466.8(425.27)$ & $3,475.3(446.52)$ & $<0.001$ \\
\hline Gender & & & & & & 0.87 \\
\hline Male & $613(48.7 \%)$ & $2,130(48.8 \%)$ & $1,757(48.5 \%)$ & $818(50.1 \%)$ & $374(49.1 \%)$ & \\
\hline Female & 647 (51.4\%) & $2,233(51.2 \%)$ & 1,866 (51.5\%) & 815 (49.9\%) & $388(50.9 \%)$ & \\
\hline Apgar 1 & $8(8-9)$ & $8(8-9)$ & $8(8-9)$ & $8(8-9)$ & $8(8-9)$ & 0.01 \\
\hline Apgar 5 & $9(9-9)$ & $9(9-9)$ & $9(9-9)$ & $9(9-9)$ & $9(9-9)$ & 0.65 \\
\hline Arterial Ph & & & & & & 0.91 \\
\hline Mean (SD) & $7.27(0.07)$ & $7.27(0.07)$ & $7.27(0.00)$ & $7.27(0.07)$ & $7.27(0.07)$ & \\
\hline Meconium & $127(10.1 \%)$ & $471(10.8 \%)$ & $446(12.3 \%)$ & $227(13.9 \%)$ & $118(15.4 \%)$ & $<0.001$ \\
\hline $\begin{array}{l}\text { Ventilator } \\
\text { support (d) }\end{array}$ & 0 & 0 & 0 & 0 & 0 & 0.21 \\
\hline \multicolumn{7}{|l|}{$\begin{array}{l}\text { Newborn sepsis/NICU } \\
\text { admit }\end{array}$} \\
\hline Suspect & $33(2.6 \%)$ & $191(4.4 \%)$ & $135(3.7 \%)$ & $70(4.3 \%)$ & $29(3.8 \%)$ & \\
\hline Proven & $3(0.24 \%)$ & $5(0.11 \%)$ & $4(0.11 \%)$ & $1(0.06 \%)$ & 0 & 0.55 \\
\hline Composite outcome $^{a}$ & $14(1.1 \%)$ & $66(1.5 \%)$ & $50(1.4 \%)$ & $27(1.7 \%)$ & $14(1.8 \%)$ & 0.65 \\
\hline NEC & $1(0.08 \%)$ & $1(0.02 \%)$ & $1(0.03 \%)$ & 0 & 0 & \\
\hline TTN & $4(0.32 \%)$ & $32(0.73 \%)$ & $24(0.66 \%)$ & $7(0.43 \%)$ & $7(0.92 \%)$ & \\
\hline $\mathrm{BDP}$ & 0 & $1(0.02 \%)$ & $1(0.03 \%)$ & $1(0.06 \%)$ & 0 & \\
\hline RDP & 7 & $38(0.87 \%)$ & $23(0.63 \%)$ & $18(1.1 \%)$ & $10(1.3 \%)$ & \\
\hline Presser use & $4(0.32 \%)$ & $7(0.16 \%)$ & $4(0.11 \%)$ & $3(0.18 \%)$ & 0 & \\
\hline PIE & 0 & 0 & $1(0.03 \%)$ & 0 & 0 & \\
\hline Hypertonicity & $1(0.08 \%)$ & 0 & $2(0.06 \%)$ & 0 & 0 & \\
\hline
\end{tabular}

Abbreviations: BDP, bronchopulmonary dysplasia; BMI, body mass index; NEC, necrotizing enterocolitis; NICU, neonatal intensive care unit; PIE, pulmonary interstitial emphysema; RDP, respiratory distress; TTN, transient tachypnea of the newborn.

Note: $q$-Value for categorical variables from chi-square test and for continuous variables from Kruskal-Wallis test across BMI categories; Data presented as $N(\%)$ Mean (SD) or Median (interquartile range).

ancludes necrotizing enterocolitis (NEC), transient tachypnea of the newborn (TTN), bronchopulmonary dysplasia (BPD), respiratory distress (RDS), presser use, pulmonary interstitial emphysema (PIE), hypertonicity.

and labor management is applied, an important consideration given the current guidelines favor an undefined prolongation of labor to avoid complications for obese patients. ${ }^{17,24}$ However, standard labor management recommendations for obese women remain elusive with no definitive data to support particular timelines or acceptable labor curves. ${ }^{17,24}$

These induction and mode of delivery results are consistent with prior studies that have described a sequential increase in induction and cesarean delivery rates with increasing maternal BMI, approaching a threefold increase in cesarean delivery for class III obese women., ${ }^{9,10}$ In this current large dataset, there were no significant differences in adverse maternal outcomes including chorioamnionitis and endomyometritis with increasing BMI class when labor management guidelines were followed, regardless of increased rates of induction and cesarean delivery among obese parturients. $3,4,9,12-15,18,19$ With standardized labor management and the contribution of contemporary protocols optimizing postoperative infection risk, ${ }^{36}$ our results suggest rates of postoperative infectious morbidity are improved and comparable across all weight classes.
However, these results should be interpreted with caution given the exclusion of all comorbidities, including those associated with obesity. Whereas other research has described a uterine atony-related hemorrhage rate approaching $5 \%$ for obese women who delivered vaginally, atonyrelated hemorrhage and the maternal composite including blood transfusion were not increased with increasing BMI in the current study. ${ }^{3,15,21,37}$ Among low-risk obese women, even with class III obesity, vaginal delivery approached $95 \%$, similar to other trials evaluating outcomes involving a lowrisk obstetrical cohort undergoing induction. ${ }^{38,39}$ It is important to note that all cesarean deliveries for nonreassuring fetal status were excluded from analysis, contributing to the low cesarean delivery rates in this analysis. These data were intentionally excluded as it may have altered time points related to labor dystocia, erroneously altering results.

Although neonatal outcomes of obese women were not significantly different from normal or overweight women, meconium passage was more frequent among the newborns of class III obese women, and newborn weight increased with maternal BMI class, consistent with prior studies describing 
lower Apgar scores and possible transitional challenge for these newborns, along with an increased risks of macrosomia. ${ }^{15,40,41}$ Any pregnancy complicated with fetal growth restriction or maternal morbidity was excluded and the remaining fetuses, although not macrosomic, were approximately $300 \mathrm{~g}$ larger when delivered from women with class III obesity. Although newborns of class III obese women had significantly higher rates of meconium passage at birth, there were no differences in Apgar scores or umbilical artery blood gas values suggestive of intrapartum fetal hypoxemia or acidosis across weight class, nor was there any differences in the composite neonatal outcome.

Current recommendations do not support antenatal surveillance or induction of labor for obese patients given the paucity of data demonstrating improved pregnancy outcomes with these interventions in the absence of comorbidities. ${ }^{3-5,8,10-15,18-21}$ This was confirmed with our analysis. When analyzing the cohort of women who underwent induction in this study, there was no difference in outcomes, including the maternal and neonatal composites according to weeks' gestation at induction across weight classes up to $40^{6 / 7}$ weeks with multivariate analysis to exclude confounders.

The publication and implementation of the labor dystocia guidelines in 2014 standardized labor management at our institution, allowing for more direct comparisons of outcomes of women with differing BMI classes, excluding the possible deleterious effects of prolonged labor or inductions. Although maternal and neonatal outcomes are improved with standardized protocols utilizing the current guidelines for labor management, cesarean delivery rates in the United States remain increased. Clinical tools to identify labor progress with obesity, such as BMI-determined labor partograms, may prove helpful in reducing cesarean rates in this cohort of women, especially when considering changes in weight gain or loss. Obese women may benefit from optimized induction or augmentation protocols related to medication dosage and interval, rather than the standard protocols currently in use for women of any BMI. All induction agents and methods may need to be evaluated to find the most effective regimen for obese patients in future studies. Although this analysis included a large cohort of low-risk obese women, further research should include a similar analysis for obese women with comorbidities to more accurately predict risk and counsel patients appropriately.

\section{Strengths and Limitations}

Study limitations include that this analysis was retrospective and derived from a large departmental database where cases may have been missed or data entered incorrectly. These data are also limited by the accuracy of times documented in the medical record. However, only $6.8 \%$ of the cases were excluded secondary to incorrect or missing BMI data. In addition, the Department of Obstetrics and Gynecology at the Baylor College of Medicine publishes evidence-based guidelines and protocols for induction of labor based on national guidelines that are available to the team of residents, fellows, and faculty at both delivery sites.
Although our institution attempts to comply with our evidence-based protocols and national standards, this may not be generalizable to other institutions, centers, or populations. Despite the discovered decrease in guideline adherence for labor management of women with increasing BMI, it is commendable that the compliance rate was overall quite high within the Baylor-affiliated centers, reflecting dedication to evidence-based training for physicians. The strengths of our study include the large study cohort of over 13,000 low-risk deliveries between 2014 and 2017 after the publication and implementation of the labor dystocia and prevention of the primary cesarean labor management guidelines were published and evidence-based protocols for induction were utilized.

\section{Conclusion}

This study describes perinatal outcomes of low-risk obese women, after adjusting for significant confounding variables that may have influenced the results of prior publications addressing adverse maternal and neonatal outcomes, especially related to labor duration. The results also demonstrate potential quality gaps surrounding protocol and guideline adherence for obese patients and possible bias when addressing this vulnerable population. Weight bias is often based on false presumptions that should be dispelled by truth and evidence. Data such as these, should be communicated and disseminated, especially in the setting of medical education.

\section{Note}

IRB approval: H-26364.

This work has not been published previously and is not under consideration for publication elsewhere.

\section{Conflict of Interest}

The authors report no conflict of interest.

\section{References}

1 Flegal KM, Carroll MD, Kit BK, Ogden CL. Prevalence of obesity and trends in the distribution of body mass index among US adults, 1999-2010. JAMA 2012;307(05):491-497

2 Ogden CL, Carroll MD, Kit BK, Flegal KM. Prevalence of childhood and adult obesity in the United States, 2011-2012. JAMA 2014; 311(08):806-814

3 Catalano PM. Management of obesity in pregnancy. Obstet Gynecol 2007;109(2 Pt 1):419-433

4 Weiss JL, Malone FD, Emig D, et al; FASTER Research Consortium. Obesity, obstetric complications and cesarean delivery rate-a population-based screening study. Am J Obstet Gynecol 2004;190 (04):1091-1097

5 Anderson NH, McCowan LM, Fyfe EM, et al; SCOPE Consortium. The impact of maternal body mass index on the phenotype of preeclampsia: a prospective cohort study. BJOG 2012;119(05):589-595

6 Salihu HM, Dunlop AL, Hedayatzadeh M, Alio AP, Kirby RS, Alexander GR. Extreme obesity and risk of stillbirth among black and white gravidas. Obstet Gynecol 2007;110(03):552-557

7 Yao R, Ananth CV, Park BY, Pereira L, Plante LA. Obesity and the risk of stillbirth: a population-based cohort study. Perinatal research consortium. Am J Obstet Gynecol 2014;210:457. e1-457.e9 
8 Salihu HM, Luke S, Alio AP, Deutsch A, Marty PJ. The impact of obesity on spontaneous and medically indicated preterm birth among adolescent mothers. Arch Gynecol Obstet 2010;282(02): 127-134

9 Hibbard JU, Gilbert S, Landon MB, et al; National Institute of Child Health and Human Development Maternal-Fetal Medicine Units Network. Trial of labor or repeat cesarean delivery in women with morbid obesity and previous cesarean delivery. Obstet Gynecol 2006;108(01):125-133

10 Chu SY, Callaghan WM, Kim SY, et al. Maternal obesity and risk of gestational diabetes mellitus. Diabetes Care 2007;30(08): 2070-2076

11 Nuthalapaty FS, Rouse DJ, Owen J. The association of maternal weight with cesarean risk, labor duration, and cervical dilation rate during labor induction. Obstet Gynecol 2004;103(03): 452-456

12 Chu SY, Kim SY, Schmid CH, et al. Maternal obesity and risk of cesarean delivery: a meta-analysis. Obes Rev 2007;8(05): 385-394

13 Wloch C, Wilson J, Lamagni T, Harrington P, Charlett A, Sheridan E. Risk factors for surgical site infection following caesarean section in England: results from a multicentre cohort study. BJOG 2012; 119(11):1324-1333

14 Duhl AJ, Paidas MJ, Ural SH, et al; Pregnancy and Thrombosis Working Group. Antithrombotic therapy and pregnancy: consensus report and recommendations for prevention and treatment of venous thromboembolism and adverse pregnancy outcomes. Am J Obstet Gynecol 2007;197(05):457.e1-457.e21

15 Gross T, Sokol RJ, King KC. Obesity in pregnancy: risks and outcome. Obstet Gynecol 1980;56(04):446-450

16 Antony KM, Hemarajata P, Chen J, et al. Generation and validation of a universal perinatal database and biospecimen repository: PeriBank. J Perinatol 2016;36(11):921-929

17 Committeee on Practice Bulletins. Obstetrics Practice Bulletin No. 156. Obesity in pregnancy. The American College of Obstetrics and Gynecology. Practice bulletin no 156. Obstet Gynecol 2015; 126:e112-e126

18 Arrowsmith S, Wray S, Quenby S. Maternal obesity and labour complications following induction of labour in prolonged pregnancy. BJOG 2011;118(05):578-588

19 Nuthalapaty FS, Rouse DJ, Owen J. The association of maternal weight with cesarean risk, labor duration, and cervical dilation rate during labor induction. Obstet Gynecol 2004;103(03): 452-456

20 Robinson BK, Mapp DC, Bloom SL, et al; Eunice Kennedy Shriver National Institute of Child Health and Human Development (NICHD) of the Maternal-Fetal Medicine Units Network (MFMU) Increasing maternal body mass index and characteristics of the second stage of labor. Obstet Gynecol 2011;118(06): 1309-1313

21 ACOG Committee on Practice Bulletins-Obstetrics. Induction of labor. ACOG Practice Bulletin No. 107. American College of Obstetricians and Gynecologists. Obstet Gynecol 2009;114:386-397

22 Flegal KM, Carroll MD, Ogden CL, Curtin LR. Prevalence and trends in obesity among US adults, 1999-2008. JAMA 2010;303(03): 235-241

23 Aune D, Saugstad OD, Henriksen T, Tonstad S. Maternal body mass index and the risk of fetal death, stillbirth, and infant death: a systematic review and meta-analysis. JAMA 2014;311(15): 1536-1546

24 Spong CY, Berghella V, Wenstrom KD, Mercer BM, Saade GR. Preventing the first cesarean delivery: summary of a joint Eunice Kennedy Shriver National Institute of Child Health and Human
Development, Society for Maternal-Fetal Medicine, and American College of Obstetricians and Gynecologists Workshop. Obstet Gynecol 2012;120(05):1181-1193

25 Lynch AM, Hart JE, Agwu OC, Fisher BM, West NA, Gibbs RS Association of extremes of prepregnancy BMI with the clinical presentations of preterm birth. Am J Obstet Gynecol 2014;210 (05):428.e1-428.e9

26 Hendler I, Goldenberg RL, Mercer BM, et al. The preterm prediction study: association between maternal body mass index and spontaneous and indicated preterm birth. Am J Obstet Gynecol 2005;192(03):882-886

27 Vahratian A, Zhang J, Troendle JF, Savitz DA, Siega-Riz AM. Maternal prepregnancy overweight and obesity and the pattern of labor progression in term nulliparous women. Obstet Gynecol 2004;104(5 Pt 1):943-951

28 Young TK, Woodmansee B. Factors that are associated with cesarean delivery in a large private practice: the importance of prepregnancy body mass index and weight gain. Am J Obstet Gynecol 2002;187(02):312-318, discussion 318-320

29 Kawakita T, Reddy UM, Landy HJ, Iqbal SN, Huang CC, Grantz KL. Indications for primary cesarean delivery relative to body mass index. Am J Obstet Gynecol 2016;215(04):515.e1-515.e9

30 American College of Obstetricians and Gynecologists Society for Maternal-Fetal Medicine. Obstetric care consensus no. 1: safe prevention of the primary cesarean delivery. Obstet Gynecol 2014;123(03):693-711

31 Antony KM, Hemarajata P, Chen J, et al. Generation and validation of a universal perinatal database and biospecimen repository: PeriBank. J Perinatol 2016;36(11):921-929

32 von Elm E, Altman DG, Egger M, Pocock SJ, Gøtzsche PC, Vandenbroucke JPSTROBE Initiative. The Strengthening the Reporting of Observational Studies in Epidemiology (STROBE) statement: guidelines for reporting observational studies. Lancet 2007;370 (9596):1453-1457

33 Geller G, Watkins PA. Addressing medical students' negative bias toward patients with obesity through ethics education. AMA J Ethics 2018;20(10):E948-E959

34 Pillutla V, Maslen H, Savulescu J. Rationing elective surgery for smokers and obese patients: responsibility or prognosis? BMC Med Ethics 2018;19(01):28

35 Phelan SM, Burgess DJ, Yeazel MW, Hellerstedt WL, Griffin JM, van Ryn M. Impact of weight bias and stigma on quality of care and outcomes for patients with obesity. Obes Rev 2015;16(04): 319-326

36 Use of prophylactic antibiotics in labor and delivery. ACOG Practice Bulletin No. 199. American College of Obstetricians and Gynecologists. Obstet Gynecol 2018;132:e103-e119

37 Blomberg M. Maternal obesity and risk of postpartum hemorrhage. Obstet Gynecol 2011;118(03):561-568

38 Sinkey RG, Blanchard CT, Szychowski JM, et al. Elective induction of labor in the 39th week of gestation compared with expectant management of low-risk multiparous women. Obstet Gynecol 2019;134(02):282-287

39 Chen HY, Grobman WA, Blackwell SC, Chauhan SP. Neonatal and maternal adverse outcomes among low-risk parous women at 3941 weeks of gestation. Obstet Gynecol 2019;134(02):288-294

40 Sewell MF, Huston-Presley L, Super DM, Catalano P. Increased neonatal fat mass, not lean body mass, is associated with maternal obesity. Am J Obstet Gynecol 2006;195(04):1100-1103

41 Hull HR, Dinger MK, Knehans AW, Thompson DM, Fields DA. Impact of maternal body mass index on neonate birthweight and body composition. Am J Obstet Gynecol 2008;198(04):416. e1-416.e6 